\title{
Residentes em psiquiatria têm documentado problemas relacionados ao álcool em pacientes ambulatoriais?
}

\author{
Do psychiatry residents document outpatients 'alcohol problems?
}

\author{
Clarissa Mendonça Corradi-Webster ${ }^{1}$, Milton Roberto Laprega ${ }^{2}$, Erikson Felipe Furtado ${ }^{3}$
}

${ }^{1}$ Mestre. Psicóloga, Departamento de Enfermagem Psiquiátrica e Ciências Humanas, Escola de Enfermagem de Ribeirão Preto, Universidade de São Paulo (USP), Ribeirão Preto, SP. ${ }^{2}$ Doutor. Professor, Departamento de Medicina Social, Faculdade de Medicina de Ribeirão Preto (FMRP), USP. ${ }^{3}$ Doutor. Professor, Departamento de Neurociências e Ciências do Comportamento, FMRP, USP.

\section{Resumo}

Introdução: O consumo de álcool por pacientes que fazem tratamento psiquiátrico pode trazer inúmeras consequências negativas. Os objetivos deste estudo foram identificar o uso problemático de álcool entre pacientes psiquiátricos ambulatoriais e verificar se esse consumo foi documentado nos prontuários por residentes de psiquiatria.

Método: Estudo descritivo, transversal, realizado em serviço ambulatorial de clínica psiquiátrica de hospital universitário localizado em Ribeirão Preto (SP). Foi utilizada uma amostra de conveniência formada por pacientes psiquiátricos ambulatoriais $(\mathrm{n}=127)$. A coleta de dados foi realizada por meio de entrevista (dados sociodemográficos e instrumento de rastreamento de abuso de álcool - CAGE) e pela leitura de todas as anotações feitas por residentes de psiquiatria nos prontuários dos pacientes entrevistados (ficha para coleta de dados do prontuário). Para a análise dos dados, foram utilizados os pontos de corte $\geq 1 \mathrm{e} \geq 2$ para o CAGE.

Resultados: Com CAGE $\geq 1,33,9 \%$ pontuaram positivo $(n=43)$ e, entre estes, $60,5 \%(n=26)$ não tinham registros em seus prontuários sobre o uso de álcool (qui-quadrado $=20,12 ; \mathrm{p}<0,001)$. Com CAGE $\geq 2,16,5 \%$ pontuaram positivo $(\mathrm{n}=21)$ e, entre estes, $38,1 \%(\mathrm{n}=8)$ não tinham registros em seus prontuários referentes ao consumo de bebidas alcó́licas (qui-quadrado $=29,10 ; \mathrm{p}<0,001$ ).

Conclusões: O número de subnotificações encontrado foi alto. Sugere-se que, no treinamento dos residentes de psiquiatria, sejam incluídos conteúdos relacionados a identificação precoce e intervenções para a prevenção de problemas relacionados ao uso de álcool.

Descritores: Diagnóstico, pacientes ambulatoriais, psiquiatria, alcoolismo.

\begin{abstract}
Introduction: Consumption of alcohol by psychiatric patients can lead to many negative consequences. The objectives of this study were to identify the problematic use of alcohol in a group of psychiatric outpatients and to verify if this consumption was documented in their medical records by psychiatry medical residents.

Methods: Descriptive and cross-sectional study, carried out at the psychiatric outpatient clinic of a university hospital located in Ribeirão Preto, state of São Paulo, Brazil. A convenience sample comprising 127 psychiatric outpatients was used. Data were collected using an interview (sociodemographic data and alcohol disorder screening instrument-CAGE) and by means of a review of all the notes written by psychiatry residents on medical charts (questionnaire for collecting data from the records). For data analysis, the CAGE cutoff points $\geq 1$ and $\geq 2$ were used.

Results: At CAGE $\geq 1,33.9 \%$ were CAGE positive $(n=43)$. Among the individuals with a positive CAGE score, $60.5 \%(n=26)$ had no record of alcohol use on their medical charts (chi-square $=20.12 ; \mathrm{p}<0.001)$. At CAGE $\geq 2,16.5 \%$ were CAGE positive $(\mathrm{n}=21)$. In $38.1 \%(\mathrm{n}=8)$ of these cases, alcohol use was not documented on their medical charts (chi-square $=29.10 ; \mathrm{p}<0.001$ ).

Conclusions: Undernotification of alcohol use was high. Topics related to early identification of and intervention for alcohol use-related problems should be included in the training of psychiatry residents.
\end{abstract}

Keywords: Diagnosis, outpatients, psychiatry, alcoholism. 


\section{Introdução}

A fim de agir preventivamente, é importante a identificação precoce dos vários padrões de consumo de álcool que podem representar risco para os indivíduos ${ }^{1}$. Em geral, os serviços de saúde frequentemente deixam de diagnosticar um grande número de usuários problemáticos de álcool, principalmente porque essas pessoas apresentam sintomas ou problemas que não parecem estar diretamente relacionados aos seus hábitos de consumo de bebidas alcoólicas.

Em um estudo nacional, foram analisadas informações sobre consumo, abuso ou dependência de álcool, obtidas de prontuários médicos dos anos 1982 e 2002, com o intuito de verificar mudanças referentes ao relato do consumo de álcool dos pacientes entre esses dois períodos. Os autores constataram a existência de subnotificação do uso de álcool em aproximadamente $50 \%$ dos prontuários médicos, percentual que se mostrou consistente nas duas amostras. Os achados mostram que os médicos de um grande hospital universitário não estavam abordando o alcoolismo de maneira adequada. Os autores apresentaram duas possíveis razões para esse achado: 1) o diagnóstico era estabelecido de maneira apropriada, mas não era relatado nos prontuários; 2) o diagnóstico não era estabelecido ${ }^{2}$. Em outro estudo realizado no Brasil em 1997, que investigou a prevalência de problemas psiquiátricos entre os pacientes de primeira vez em um hospital universitário, o alcoolismo foi diagnosticado em $23,2 \%$ dos casos, dos quais somente $7,4 \%$ haviam sido encaminhados para tratamento ${ }^{3}$.

Outra complicação diz respeito ao fato de os pacientes geralmente serem atendidos por profissionais que têm dificuldade em fazer perguntas sobre o uso de álcool e, portanto, em diagnosticar problemas relacionados ao álcool. Mesmo com os instrumentos de rastreamento exaustivamente estudados que estão disponíveis para auxiliar os profissionais a estabelecer um diagnóstico, em uma amostra estadunidense de psiquiatras (a especialidade médica historicamente responsável por lidar com transtornos relacionados ao álcool), observou-se que menos de $20 \%$ relataram o uso de instrumentos formais de rastreamento do uso de álcool ${ }^{4}$.

A importância do diagnóstico e da intervenção precoces é ainda maior no caso dos pacientes psiquiátricos, já que o álcool pode prejudicar a adesão ao tratamento, interagir com a medicação e piorar o prognóstico da doença. Além disso, de acordo com a Organização Mundial da Saúde (OMS), a probabilidade de dependência de álcool durante a vida para um indivíduo com transtorno psiquiátrico é significativamente maior do que para a população em geral, 22 e $14 \%$, respectivamente 5 .

Um estudo realizado com pacientes afetados por transtornos de humor utilizando diferentes questionários para avaliar transtornos relacionados ao uso de álcool detectou uma prevalência entre 20 e $30 \%$, dependendo do instrumento utilizado. Os autores concluíram que os transtornos relacionados ao uso de álcool e ao consumo de álcool de risco são frequentes em pacientes com transtornos de humor; contudo, não haviam sido registrados diagnósticos de uso de álcool em seus prontuários ${ }^{6}$.

Muitos profissionais de saúde mencionam que não avaliam o uso de álcool porque não sabem como lidar com o problema, não têm conhecimento sobre o uso de instrumentos de rastreamento rápidos ou não refletiram sobre a influência do uso problemático de álcool sobre a saúde do paciente ${ }^{1,7}$. Isso indica que o pequeno número de diagnósticos de problemas relacionados ao álcool pode ser explicado pelas deficiências no treinamento e na formação desses profissionais. Devido à alta incidência de transtornos relacionados ao uso de substâncias entre os pacientes que procuram tratamento psiquiátrico, é importante que todos os residentes de psiquiatria recebam treinamento sobre rastreamento, diagnóstico e tratamento de pacientes ambulatoriais com esses problemas ${ }^{8}$.

\section{Objetivo}

Esta comunicação breve tem o objetivo de descrever o uso problemático de álcool entre pacientes de um serviço ambulatorial de psiquiatria e verificar a ocorrência da notificação de diagnóstico de uso de álcool nos prontuários desses pacientes.

\section{Método}

Este é um estudo transversal realizado no Serviço Ambulatorial de Clínica Psiquiátrica (SACP) do Hospital das Clínicas da Faculdade de Medicina de Ribeirão Preto, Universidade de São Paulo (USP), Ribeirão Preto (SP). Foi utilizada uma amostra de conveniência de uma população ambulatorial, selecionada de acordo com critérios de adesão, sem um regime fixo de ordenação de inclusão e independente de qualquer interferência dos pesquisadores assistentes. Os critérios de inclusão foram os seguintes: ter mais de 18 anos, frequentar o SACP após passar por triagem e avaliação segundo os diagnósticos da Classificação Internacional de Doenças $-10^{\mathrm{a}}$ revisão (CID-10) e após o estabelecimento de plano terapêutico, ser capaz de fornecer informações confiáveis e concordar em participar do estudo. Os pacientes que precisavam de intervenção urgente foram excluídos.

Os dados foram coletados entre janeiro de 2000 e abril de 2004 pelos pesquisadores assistentes (estudantes de medicina e psicólogos). Todos os pesquisadores assistentes foram treinados em técnicas de entrevista, uso de instrumentos de rastreamento e avaliação para a identificação de transtornos relacionados ao uso de álcool através dos critérios diagnósticos da CID-10. A coleta de dados foi regularmente supervisionada pelo terceiro autor. Os entrevistadores iam ao SACP durante os horários de atendimento dos pacientes, e, no final das consultas, o médico residente explicava os objetivos da pesquisa aos pacientes e perguntava se eles gostariam de participar. Se os pacientes concordassem, o residente saía do consultório e convidava os pesquisadores assistentes a iniciar a coleta de 
dados. Os entrevistadores liam, juntamente com o paciente, o termo de consentimento livre e esclarecido, verificavam se o paciente realmente desejava participar e então iniciavam a entrevista para coleta de dados sociodemográficos. Posteriormente, o CAGE era aplicado.

Após a entrevista, os pesquisadores assistentes obtinham o prontuário dos pacientes e, como as anotações médicas não seguem um formato padronizado, liam todas as anotações feitas pelos residentes de psiquiatria desde a entrada do paciente no serviço psiquiátrico, incluindo anamnese, anotações das consultas de acompanhamento e anotações sobre as discussões de caso realizadas com os supervisores dos residentes. Este estudo não teve como objetivo avaliar a qualidade das anotações, mas verificar a presença de referências ao consumo de álcool. Portanto, se houvesse qualquer menção ao uso de álcool, os pesquisadores assistentes marcavam "sim" em seu protocolo de pesquisa. Os pesquisadores também registravam o diagnóstico da última consulta anotado nos prontuários dos pacientes.

Com relação à análise dos dados do CAGE, foi avaliado o desempenho do CAGE na identificação do uso problemático de álcool nesta amostra. Os melhores valores do CAGE para sensibilidade e especificidade foram encontrados com o ponto de corte $\geq 1$ (sensibilidade $=100 \%$; especificidade $=73,7 \%)^{9}$. Contudo, a literatura sugere o uso de pontos de corte do CAGE $\geq 1 \mathrm{e} \geq 2$ para análise de dados. Em um estudo realizado com pacientes psiquiátricos, o ponto de corte igual a 1 forneceu um melhor reconhecimento dos sujeitos afetados por transtorno de humor com um possível transtorno de uso de álcool. Os autores consideraram que esse ponto de corte poderia aumentar o risco de casos falso-positivos; portanto, os dados também foram avaliados para um ponto de corte igual a $2^{6}$. Em decorrência dessas diferenças, decidiu-se utilizar os dois pontos de corte, $\geq 1 \mathrm{e} \geq 2$. O software Epi-Info para Windows foi utilizado para o gerenciamento dos dados e para realizar a análise estatística (análise descritiva e testes do qui-quadrado). Valor de alfa de 0,5 e intervalo de confiança de $95 \%$ foram adotados.

Este projeto de pesquisa foi aprovado pelo Comitê de Ética da Faculdade de Medicina de Ribeirão Preto, USP. As entrevistas foram realizadas após os pacientes assinarem o termo de consentimento livre e esclarecido para sujeitos voluntários, de acordo com os critérios éticos da resolução $\mathrm{n}^{\circ} 196$ de 10 de outubro de 1996. Após a entrevista, todos os participantes receberam orientações sobre uso moderado de álcool e sobre situações em que deveriam evitar o consumo de bebidas alcoólicas.

\section{Resultados}

\section{Características da amostra}

A amostra foi formada por 127 pacientes, sendo $69,3 \%$ (n $=88$ ) deles mulheres. A idade média era de 42,9 anos (desvio padrão $=13,2$; idade mínima $=18$ e idade máxima $=77$ anos $)$.
A maioria dos participantes declarou-se branco $(74,8 \% ; \mathrm{n}=$ 95), e 66 (52,0\%) pacientes não tinham parceiros. A maioria tinha até 8 anos de estudo $(57,5 \% ; n=73)$. A renda familiar para mais da metade $(57,5 \% ; n=73)$ dos entrevistados variou entre um e cinco salários mínimos, e a maioria deles $(61,4 \% ; n=78)$ praticava alguma religião. Os diagnósticos incluíram esquizofrenia $(26,8 \% ; \mathrm{n}=34)$, transtorno afetivo bipolar $(23,6 \% ; n=30)$, episódio depressivo $(25,9 \% ; n=33)$, transtorno fóbico-ansioso $(2,4 \% ; \mathrm{n}=3)$ e outros transtornos de ansiedade $(21,3 \% ; n=27)$.

\section{Identificação de uso problemático de álcool}

Adotando-se o ponto de corte $\geq 1$ para o CAGE, foram encontrados escores positivos para $33,9 \%(n=43)$ dos sujeitos e escores negativos para $66,1 \%(n=84)$. Com relação à identificação e à notificação de consumo problemático de álcool realizadas pelos residentes para casos positivos, $60,5 \%(\mathrm{n}=$ 26) não tinham registro de uso de álcool em seus prontuários e, nos prontuários restantes, $39,5 \%(\mathrm{n}=17)$, o uso de álcool foi mencionado (qui-quadrado $=20,12 ; \mathrm{p}<0,001$ ).

Adotando-se o ponto de corte $\geq 2$ para o CAGE, foram encontrados escores positivos para $16,5 \%(n=21)$ dos sujeitos e escores negativos para $83,5 \%(n=106)$. Os prontuários de pacientes positivos não mencionaram consumo de álcool em $38,1 \%(\mathrm{n}=8)$ dos casos, e o uso de álcool foi mencionado em $61,9 \%(n=13)($ qui-quadrado $=29,10 ; p<0,001)$.

A amostra total $(n=127)$ incluiu 104 pacientes para os quais não havia nenhuma menção de consumo de álcool nos prontuários. Vinte e seis (25\%) desses 104 indivíduos tiveram escores positivos no CAGE (considerando os resultados encontrados pelo ponto de corte $\geq 1$ ).

\section{Discussão}

Nos últimos anos, a literatura tem dado atenção à importância da avaliação do uso de álcool como uma oportunidade para realizar intervenções educacionais e preventivas, esclarecer o consumo de álcool de risco e fornecer informações sobre como reduzi-lo, prevenindo possíveis problemas relacionados ao uso de álcool ${ }^{1}$.

Por essas razões, vários instrumentos (p. ex.: CAGE, Alcohol Use Disorders Identification Test - AUDIT) têm sido desenvolvidos para auxiliar a identificação. Tais instrumentos caracterizam-se por aplicabilidade fácil e rápida e exigência de treinamento profissional mínimo ${ }^{1}$. Com base na literatura, é possível verificar que questões referentes à quantidade e à frequência, impressões clínicas e dados laboratoriais não têm um desempenho tão bom quanto os instrumentos estruturados, e a escolha do instrumento de rastreamento deve levar em consideração sua precisão através do espectro de problemas relacionados ao álcool ${ }^{10}$.

As perguntas do CAGE parecem mais apropriadas para identificar os pacientes com abuso e dependência de álcool, 
enquanto que o AUDIT é mais sensível a bebedores de risco ${ }^{10}$. A literatura sugere que, na prática, os profissionais usam o CAGE com mais frequência do que o AUDIT ${ }^{11}$. Isso pode ser explicado pela quantidade de perguntas de cada instrumento (CAGE - quatro perguntas; AUDIT - 10 perguntas), levandose em consideração que os profissionais geralmente mencionam a falta de tempo como uma razão para a subnotificação do uso problemático de álcool ${ }^{1}$.

Apesar da disponibilidade desses instrumentos, em nosso estudo, observou-se que entre $38,1 \%(\geq 2)$ e $60,5 \%(\geq 1)$ dos casos positivos não apresentavam dados sobre o uso de álcool em seus prontuários. As taxas de identificação foram mais altas ao se utilizar um ponto de corte $\geq 2$. Isso possivelmente se deve ao fato desses pacientes apresentarem mais sintomas do que os identificados através do ponto de corte $\geq 1$. Em um estudo italiano realizado com pacientes afetados por transtornos de humor, o diagnóstico de transtorno do uso de álcool não havia sido registrado em nenhum dos prontuários, mesmo para aqueles pacientes que apresentavam o transtorno no momento da avaliação ${ }^{6}$. Esses dados destacam uma questão importante: os psiquiatras não estão reconhecendo e tratando os transtornos do uso de álcool.

Além da falta de tempo, essa deficiência pode ser causada pelo fato de que os profissionais de saúde não são suficientemente preparados no que se refere a questões que envolvam o uso de álcool para poderem aconselhar seus pacientes. Portanto, há a necessidade de dar mais atenção para questões educacionais. O presente estudo utilizou informações obtidas de prontuários médicos preenchidos por residentes de psiquiatria durante o primeiro e o segundo ano de residência. Os residentes de psiquiatria recebem treinamento teórico padrão, assim como supervisão prática abrangente para cada consulta feita por um psiquiatra experiente, de acordo com uma abordagem amplamente aceita para a avaliação psiquiátrica.

Um estudo nacional sugeriu que não há um número suficiente de horas no currículo dos estudantes de medicina para o ensino e o treinamento de temas relacionados à identificação e ao tratamento dos transtornos do uso de álcool ${ }^{2}$. No Reino Unido, observou-se que poucos profissionais de saúde mental tinham treinamento e/ou experiência clínica suficientes que os preparassem para intervir em casos de diagnóstico duplo ${ }^{12}$. Com relação aos benefícios do treinamento, um curso de treinamento de 16 horas para os profissionais de atenção primária à saúde sobre instrumentos de rastreamento e intervenções breves para problemas relacionados ao álcool teve um efeito positivo significativo no nível de consciência sobre problemas com álcool e na confiança dos profissionais ao lidar com essas questões ${ }^{13}$.

Com o intuito de preparar melhor os estudantes e os médicos para identificar, prevenir e tratar os problemas relacionados ao uso de álcool, o currículo das faculdades de medicina e a educação médica continuada deveriam abordar essas questões seriamente ${ }^{14}$. O Hospital Geral de Massachusetts e o Hospital
McLean oferecem um exemplo de programa de residência em psiquiatria em que os residentes recebem treinamento em psiquiatria da adição em diferentes locais de tratamento como parte do programa de residência de 4 anos $^{8}$.

Foram identificadas quatro possíveis limitações neste estudo. A primeira diz respeito à aceitação em participar do estudo. $\mathrm{O}$ número de pacientes que não aceitaram participar do estudo não foi registrado, e pode-se supor que algumas pessoas, estando cientes do objetivo do estudo, recusaram-se a participar para evitar perguntas indesejadas sobre uso de álcool. Se essa hipótese estiver correta, o número real de usuários problemáticos de álcool pode ter sido subestimado.

A segunda limitação está relacionada ao fato de os sujeitos serem pacientes que já haviam recebido um diagnóstico e um plano terapêutico, sendo acompanhados no ambulatório. Se usuários de primeira vez tivessem sido selecionados, talvez fosse possível encontrar um número maior de usuários problemáticos de álcool, considerando-se que os indivíduos com problemas relacionados ao álcool têm mais dificuldades em aderir ao tratamento.

O uso de dados dos prontuários para a obtenção de informações sobre a identificação do uso de álcool poderia ser outra limitação, com a possibilidade de que a identificação e a intervenção tenham ocorrido, mas não tenham sido documentadas. Embora essa possibilidade possa representar alguns casos de subnotificação, acredita-se que o uso problemático de álcool permanece subdiagnosticado em muitos casos, dado que é corroborado pela literatura.

A última limitação se refere ao uso do CAGE, que identifica os problemas relacionados ao álcool durante toda a vida. No presente estudo, alguns pacientes podem ter tido resultados positivos devido ao uso de álcool no passado, anteriormente ao tratamento, e isso pode representar a ausência de notificação pelo médico. Contudo, mesmo tendo sido esse o caso, o rastreamento e a documentação de uso problemático de álcool no passado é importante, já que os pacientes com esse histórico enfrentam riscos mais altos de recaídas e ainda se beneficiariam de intervenções preventivas.

\section{Conclusão}

Este estudo ressalta a questão do subdiagnóstico de problemas com álcool por residentes de psiquiatria. A subnotificação pode refletir a tendência desses profissionais de registrar informações clínicas consideradas relevantes somente para a consulta. Sugere-se que seja dada mais ênfase à identificação e à intervenção precoces de problemas relacionados ao álcool no treinamento de residentes de psiquiatria, abordando-se o estigma, as atitudes e as deficiências do conhecimento profissional. É importante enfatizar o fato de que tratamentos psicológicos e farmacológicos podem ser eficientes na redução do consumo de álcool. 


\section{Agradecimento}

Os autores gostariam de agradecer a Martin Webster pela versão em inglês deste artigo.

\section{Referências}

1. Babor TF, Higgins-Biddle JC, Saunders JB, Monteiro M. AUDIT: The Alcohol Use Disorders Identification Test: guidelines for use in primary care. 2nd ed. Geneva: World Health Organization; 2001.

2. Pechansky F, Genro VK, Diemen LV, Kessler FHP, Silveira-Santos RAP. References to alcohol consumption and alcoholism in medical records of a general hospital of Porto Alegre, Brazil - a comparison between samples with a 20-year gap. Subst Abus. 2004;25(2):29-34.

3. Smaira SI, Torres AR, Cacace LA, Giovanetti FA, Kerr-Côrrea F. Identificação de sintomas e queixas psiquiátricas no primeiro atendimento clínico de um hospital universitário [Identification of symptoms and psychiatric complaints in the first clinical attendance of a university hospital]. Inf Psiquatr. 1997;16(3):88-91.

4. Friedmann PD, McCullough D, Chin MH, Saitz R. Screening and intervention for alcohol problems: a national survey of primary care physicians and psychiatrists. J Gen Intern Med. 2000;15:84-91.

5. World Health Organization (WHO). Neuroscience of psychoactive substance use and dependence. Genebra: WHO; 2004.
6. Agabio R, Marras P, Gessa GL, Carpiniello B. Alcohol use disorders, and at-risk drinking in patients affected by a mood disorder, in Cagliari, Italy: sensitivity and specificity of different questionnaires. Alcohol Alcohol. 2007;42(6):575-81.

7. Kerr-Côrrea F, Smaira SI, Torres AR, Rossini R. É possível melhorar o diagnóstico de alcoolismo? Avaliação do ensino de psiquiatria através de interconsultas em enfermarias de um hospital universitário. Rev ABPAPAL. 1989;11(2):81-5.

8. Iannucci R, Sanders K, Greenfield SF. A 4-year curriculum on substance use disorders for psychiatry residents. Acad Psychiatry. 2009;33(1):60-6.

9. Corradi-Webster CM, Laprega MR, Furtado EF. Performance assessment of CAGE screening test among psychiatric outpatients. Rev Lat Am Enfermagem. 2005;13:1213-18.

10. Fiellin DA, Reid MC, O'Connor PG. Screening for alcohol problems in primary care: a systematic review. Arch Intern Med. 2000;160:1977-89.

11. Bradley KA, Kivlahan DR, Zhou XH, Sporleder JL, EplerAJ, McCormick KA, et al. Using alcohol screening results and treatment history to assess the severity of at-risk drinking in Veterans Affairs primary care patients. Alcohol Clin Exp Res. 2004;28(3):448-55.

12. Hughes E, Wanigaratne S, Gournay K, Johnson S, Thornicroft G, Finch E, et al. Training in dual diagnosis interventions (the COMO Study): randomised controlled trial. BMC Psychiatry. 2008;8(12):1-9.

13. Furtado EF, Corradi-Webster CM, Laprega MR. Implementing brief interventions for alcohol problems in the public health system in the region of Ribeirão Preto, Brazil: evaluation of the PAI-PAD training model. Nord Stud Alcohol Drugs. 2008;25(6):539-51.

14. Frost-Pineda K, VanSusteren T, Gold MS. Are physicians and medical students prepared to educate patients about alcohol consumption? J Addict Dis. 2004;23(2):1-13. 